\title{
Effective action of bosonic string theory at order $\alpha^{22}$
}

\author{
Mohammad R. Garousi ${ }^{\mathrm{a}}$ \\ Department of Physics, Faculty of Science, Ferdowsi University of Mashhad, P.O. Box 1436, Mashhad, Iran
}

Received: 13 August 2019 / Accepted: 29 September 2019 / Published online: 8 October 2019

(C) The Author(s) 2019

\begin{abstract}
Recently, it has been shown that the gauge invariance requires the minimum number of independent couplings for $B$-field, metric and dilaton at order $\alpha^{\prime 2}$ to be 60 . In this paper we fix the corresponding 60 parameters in string theory by requiring the couplings to be invariant under the global T-duality transformations. The Riemann cubed terms are exactly the same as the couplings that have been found by the S-matrix calculations.
\end{abstract}

\section{Introduction}

String theory is a quantum theory of gravity with a finite number of massless fields and a tower of infinite number of massive fields reflecting the stringy nature of the gravity. An efficient way to study different phenomena in this theory is to use an effective action which includes only massless fields. The effects of the massive fields appear in the action as the higher derivatives of the massless fields. This effective action may be found by imposing various symmetries/dualities in the string theory. There are various gauge symmetries in the effective actions which are corresponding to the various massless fields, e.g., the diffeomorphism symmetry corresponds to the metric and the gauge symmetry corresponds to the Kalb-Ramond field or $B$-field. In the bosonic string theory which has only metric, dilaton and $B$-field, they are the only local symmetries of the effective action. Imposing only these symmetries, one finds the effective action has three couplings at order $\alpha^{\prime 0}$ (two-derivative order), has 8 couplings at order $\alpha^{\prime}$ (four-derivative order) up to field redefinitions [1], has 60 couplings at order $\alpha^{\prime 2}$ (six-derivative order) [2] and so on. The gauge symmetries, however, can not determine the coefficients of the couplings. These parameters may be found by $\mathrm{S}$-matrix calculations $[3,4]$, by sigma-model calculations [5-7] or by imposing global symmetries of the string theory in which we are interested.

a e-mail: garousi@um.ac.ir
One of the global symmetries of the string theory is Tduality $[8,9]$. This duality like the above gauge symmetries may be imposed at the action level to fix the parameters of the effective action at any order of $\alpha^{\prime}$. One approach for imposing this symmetry is the double field theory (DFT) [10-14] in which the $D$-dimensional effective action is extended to $2 D$ space. In this theory, the gauge transformations are deformed to receive $\alpha^{\prime}$-corrections whereas the T-duality symmetry is imposed without deformation simply by writing the couplings as $O(D, D)$ scalars [14-18]. Another approach is to reduce the $D$-dimensional gauge invariant theory on a circle and impose the T-duality symmetry by constraining the couplings in the $(D-1)$-dimensional spacetime to be $Z_{2}$ scalars [19] where $Z_{2}$-group is the Buscher rules [20,21] plus their $\alpha^{\prime}$-deformations [22-24]. Using this approach for the case that $B$-field is zero, the known gravity and dilaton couplings in the effective actions at orders $\alpha^{\prime}, \alpha^{\prime 2}, \alpha^{\prime 3}$ have been found in $[25,26]$, up to some overall factors. Moreover, when $B$-field is non-zero, the known couplings at order $\alpha^{\prime}$ and their corresponding corrections to the Buscher rules have been found in [27]. In this paper, we are going to use this approach to find the couplings at order $\alpha^{\prime 2}$ for the case that $B$-field is non-zero. These couplings, except its Riemann cubed couplings, have not been found by any other methods in string theory.

It is known that the effective action at order $\alpha^{\prime 2}$ depends on the scheme that one uses for the effective action at order $\alpha^{\prime}$ [28]. In the T-duality approach, this is reflected to the Tduality transformations at order $\alpha^{\prime}$. It has been observed in [27] that the T-duality transformations at order $\alpha^{\prime}$ depends on the scheme that one uses for the effective action at order $\alpha^{\prime}$. The T-duality transformation corresponding to the effective action at order $\alpha^{\prime}$ which has only first time derivative [29], is given in [24]. The T-duality transformations at order $\alpha^{\prime}$ corresponding to the effective action at order $\alpha^{\prime}$ in an arbitrary scheme have been found in [27]. In this paper we are going to find the effective action at order $\alpha^{2}$ that correspond to the 
effective action at order $\alpha^{\prime}$ which has minimum number of couplings [1].

The outline of the paper is as follows: In Sect. 2, we write the known minimum number of couplings at orders $\alpha^{\prime}$ and $\alpha^{\prime 2}$ that the gauge symmetry can fix up to field redefinitions. In Sect. 3, we impose the T-duality symmetry on the gauge invariant couplings to find their corresponding parameter. The calculations at order $\alpha^{\prime}$ have been already done in [27]. That calculations produce the known couplings in the literature and the corresponding T-duality transformations. The calculations at order $\alpha^{\prime 2}$ are new. We have found both the effective action and the corresponding T-duality transformations. However, since the expressions for the T-duality transformations are very lengthy we will write only the effective action (see (40)). We have found that there are only 27 nonzero couplings in the effective action at order $\alpha^{\prime 2}$. Two of them have already been found by the S-matrix calculations [30]. All other terms are new couplings that the T-duality constraint produces. In Sect. 4, we briefly discuss our results.

\section{Gauge invariance constraint}

The effective action of string theory has a double expansions. One expansion is the genus expansion which includes the classical sphere-level and a tower of quantum effects. The other one is a stringy expansion which is an expansion in terms of higher-derivative or $\alpha^{\prime}$-expansion. The classical effective action has the following $\alpha^{\prime}$-expansion in the string frame:

$$
\begin{aligned}
\mathbf{S}_{\mathrm{eff}} & =\sum_{n=0}^{\infty} \alpha^{\prime n} \mathbf{S}_{n}=\mathbf{S}_{0}+\alpha^{\prime} \mathbf{S}_{1}+\alpha^{\prime 2} \mathbf{S}_{2}+\cdots \\
\mathbf{S}_{n} & =-\frac{2}{\kappa^{2}} \int d^{D} x \sqrt{-G} e^{-2 \Phi} \mathcal{L}_{n}
\end{aligned}
$$

The effective action must be invariant under the coordinate transformations and under the $B$-field gauge transformations. So the metric $G_{\mu \nu}$, the antisymmetric $B$-field and dilaton $\Phi$ must appear in the Lagrangian $\mathcal{L}_{n}$ trough their field strengths and their covariant derivatives. This requires the effective action at order $\alpha^{\prime 0}$ to have the following couplings:

$\mathcal{L}_{0}=a_{1} R+a_{2} \nabla_{\alpha} \Phi \nabla^{\alpha} \Phi+a_{3} H_{\alpha \beta \gamma} H^{\alpha \beta \gamma}$

where $a_{1}, a_{2}, a_{3}$ are three parameters which can not be fixed by the gauge invariance constraints.

At higher orders of $\alpha^{\prime}$, one has the freedom of using field redefinitions and the Bianchi identities. As a result, there are no unique form for the couplings, even the number of couplings are not unique at the higher orders of $\alpha^{\prime}$. There are however, schemes in which the number of couplings are minimum. It has been shown in [1] that the minimum number of couplings at order $\alpha^{\prime}$ is 8 . These eight couplings can also be written in different schemes. In one particular such scheme, the couplings can be written as [2]

$\mathcal{L}_{1}=\mathcal{L}_{1}^{1}+\mathcal{L}_{1}^{2}$

where $\mathcal{L}_{1}^{1}$ includes the minimum number of couplings which do not include the dilaton, i.e., ,

$$
\begin{aligned}
\mathcal{L}_{1}^{1}= & b_{1} R_{\alpha \gamma \beta \delta} R^{\alpha \beta \gamma \delta}+b_{2} H_{\alpha}{ }^{\delta \epsilon} H^{\alpha \beta \gamma} H_{\beta \delta}{ }^{\zeta} H_{\gamma \epsilon \zeta} \\
& +b_{3} H_{\alpha \beta}{ }^{\delta} H^{\alpha \beta \gamma} H_{\gamma}{ }^{\epsilon \zeta} H_{\delta \epsilon \zeta}+b_{4} H_{\alpha}{ }^{\delta \epsilon} H^{\alpha \beta \gamma} R_{\beta \delta \gamma \epsilon}
\end{aligned}
$$

and $\mathcal{L}_{1}^{2}$ includes the other couplings which all include nontrivially the dilaton, i.e., ,

$$
\begin{aligned}
\mathcal{L}_{1}^{2}= & b_{5} H_{\beta \gamma \delta} H^{\beta \gamma \delta} \nabla_{\alpha} \Phi \nabla^{\alpha} \Phi+b_{6} H_{\alpha}{ }^{\gamma \delta} H_{\beta \gamma \delta} \nabla^{\alpha} \Phi \nabla^{\beta} \Phi \\
& +b_{7} H_{\alpha}{ }^{\gamma \delta} H_{\beta \gamma \delta} \nabla^{\beta} \nabla^{\alpha} \Phi+b_{8} \nabla_{\alpha} \Phi \nabla^{\alpha} \Phi \nabla_{\beta} \Phi \nabla^{\beta} \Phi
\end{aligned}
$$

where $b_{1}, \ldots, b_{8}$ are eight parameters which can not be fixed by the gauge invariance constraints.

At order $\alpha^{\prime 2}$, the minimum number of couplings is 60 . In one particular minimal scheme in which there is no $R, R_{\mu \nu}, \nabla_{\mu} H^{\mu \alpha \beta}, \nabla_{\mu} \nabla^{\mu} \Phi$, the couplings are [2]

$\mathcal{L}_{2}=\mathcal{L}_{2}^{1}+\mathcal{L}_{2}^{2}$

where $\mathcal{L}_{2}^{1}$ has the minimum number of couplings in which the dilaton does not appear, i.e., ,

$$
\begin{aligned}
& \mathcal{L}_{2}^{1}=c_{1} R_{\alpha}{ }^{\epsilon} \gamma^{\zeta} R^{\alpha \beta \gamma \delta} R_{\beta \zeta \delta \epsilon}+c_{2} R_{\alpha \beta}{ }^{\epsilon \zeta} R^{\alpha \beta \gamma \delta} R_{\gamma \epsilon \delta \zeta} \\
& +c_{3} H_{\alpha}{ }^{\delta \epsilon} H^{\alpha \beta \gamma} H_{\beta \delta}{ }^{\zeta} H_{\gamma}{ }^{l \kappa} H_{\epsilon l}{ }^{\mu} H_{\zeta \kappa \mu} \\
& +c_{4} H_{\alpha \beta}{ }^{\delta} H^{\alpha \beta \gamma} H_{\gamma}{ }^{\epsilon \zeta} H_{\delta}{ }^{\imath \kappa} H_{\epsilon \zeta}{ }^{\mu} H_{l \kappa \mu} \\
& +c_{5} H_{\alpha \beta}{ }^{\delta} H^{\alpha \beta \gamma} H_{\gamma}{ }^{\epsilon \zeta} H_{\delta \epsilon}{ }^{l} H_{\zeta}{ }^{\kappa \mu} H_{\iota \kappa \mu} \\
& +c_{6} H_{\alpha}{ }^{\delta \epsilon} H^{\alpha \beta \gamma} H_{\beta}{ }^{\zeta \iota} H_{\delta \zeta}{ }^{\kappa} R_{\gamma \epsilon \iota \kappa} \\
& +c_{7} H_{\alpha}{ }^{\delta \epsilon} H^{\alpha \beta \gamma} R_{\beta}{ }^{\zeta}{ }^{l} R_{\gamma \zeta \epsilon l} \\
& +c_{8} H_{\alpha \beta}{ }^{\delta} H^{\alpha \beta \gamma} H_{\epsilon \zeta}{ }^{\kappa} H^{\epsilon \zeta \iota} R_{\gamma \iota \delta \kappa} \\
& +{ }_{9} H^{\alpha \beta \gamma} H^{\delta \epsilon \zeta} R_{\alpha \beta \delta}{ }^{\iota} R_{\gamma \iota \epsilon \zeta} \\
& +c_{10} H_{\alpha}{ }^{\delta \epsilon} H^{\alpha \beta \gamma} R_{\beta}{ }^{\zeta}{ }^{l} R_{\gamma l \epsilon \zeta} \\
& +c_{11} H_{\alpha}{ }^{\delta \epsilon} H^{\alpha \beta \gamma} R_{\beta^{\zeta}} \gamma^{\iota} R_{\delta \zeta \epsilon \iota} \\
& +c_{12} H_{\alpha \beta}{ }^{\delta} H^{\alpha \beta \gamma} R_{\gamma}{ }^{\epsilon \zeta \imath} R_{\delta \zeta \epsilon l} \\
& +c_{13} H_{\alpha \beta}{ }^{\delta} H^{\alpha \beta \gamma} H_{\gamma}{ }^{\epsilon \zeta} H_{\epsilon}{ }^{\iota \kappa} R_{\delta \iota \zeta \kappa} \\
& +c_{14} H_{\alpha}{ }^{\delta \epsilon} H^{\alpha \beta \gamma} H_{\beta \delta}{ }^{\zeta} H_{\gamma}{ }^{\imath \kappa} R_{\epsilon \iota \zeta \kappa} \\
& +c_{15} H_{\alpha \beta}{ }^{\delta} H^{\alpha \beta \gamma} H_{\gamma}{ }^{\epsilon \zeta} H_{\delta}{ }^{\iota \kappa} R_{\epsilon l \zeta \kappa} \\
& +c_{16} H_{\alpha}{ }^{\delta \epsilon} H^{\alpha \beta \gamma} \nabla_{\iota} H_{\delta \epsilon \zeta} \nabla^{\iota} H_{\beta \gamma}{ }^{\zeta} \\
& +c_{17} H_{\alpha}{ }^{\delta \epsilon} H^{\alpha \beta \gamma} \nabla_{\zeta} H_{\gamma \epsilon l} \nabla^{\iota} H_{\beta \delta^{\zeta}} \\
& +c_{18} H_{\alpha}{ }^{\delta \epsilon} H^{\alpha \beta \gamma} \nabla_{\iota} H_{\gamma \epsilon \zeta} \nabla^{\iota} H_{\beta \delta^{\zeta}} \\
& +c_{19} H_{\alpha \beta}{ }^{\delta} H^{\alpha \beta \gamma} \nabla_{\zeta} H_{\delta \epsilon \iota} \nabla^{\iota} H_{\gamma}{ }^{\epsilon \zeta} \\
& +c_{20} H_{\alpha \beta}{ }^{\delta} H^{\alpha \beta \gamma} \nabla_{\iota} H_{\delta \epsilon \zeta} \nabla^{\iota} H_{\gamma}{ }^{\epsilon \zeta}
\end{aligned}
$$

and $\mathcal{L}_{2}^{2}$ has the other couplings which all include derivatives of the dilaton, i.e., ,

$$
\begin{aligned}
\mathcal{L}_{2}^{2}= & c_{21} H_{\beta}{ }^{\epsilon \zeta} H^{\beta \gamma \delta} H_{\gamma \epsilon}{ }^{\imath} H_{\delta \zeta \iota} \nabla_{\alpha} \Phi \nabla^{\alpha} \Phi \\
& +c_{22} R_{\beta \gamma \delta \epsilon} R^{\beta \gamma \delta \epsilon} \nabla_{\alpha} \Phi \nabla^{\alpha} \Phi
\end{aligned}
$$




$$
\begin{aligned}
& +c_{23} H_{\beta}{ }^{\epsilon \zeta} H^{\beta \gamma \delta} R_{\gamma \in \delta \zeta} \nabla_{\alpha} \Phi \nabla^{\alpha} \Phi \\
& +c_{24} H_{\alpha}{ }^{\gamma \delta} H_{\beta}{ }^{\epsilon \zeta} H_{\gamma \epsilon}{ }^{\iota} H_{\delta \zeta \iota} \nabla^{\alpha} \Phi \nabla^{\beta} \Phi \\
& +c_{25} R_{\alpha}{ }^{\gamma \delta \epsilon} R_{\beta \delta \gamma \epsilon} \nabla^{\alpha} \Phi \nabla^{\beta} \Phi \\
& +c_{26} H_{\alpha}{ }^{\gamma \delta} H_{\beta}{ }^{\epsilon \zeta} R_{\gamma \in \delta \zeta} \nabla^{\alpha} \Phi \nabla^{\beta} \Phi \\
& +c_{27} H_{\gamma \delta \epsilon} H^{\gamma \delta \epsilon} \nabla^{\alpha} \Phi \nabla_{\beta} \nabla_{\alpha} \Phi \nabla^{\beta} \Phi \\
& +c_{28} H_{\alpha}{ }^{\gamma \delta} H_{\beta}{ }^{\epsilon \zeta} H_{\gamma \epsilon}{ }^{\iota} H_{\delta \zeta \iota} \nabla^{\beta} \nabla^{\alpha} \Phi \\
& +c_{29} H_{\alpha}{ }^{\gamma \delta} H_{\beta}{ }^{\epsilon \zeta} H_{\gamma \delta}{ }^{\iota} H_{\epsilon \zeta \iota} \nabla^{\beta} \nabla^{\alpha} \Phi \\
& +c_{30} H_{\alpha}{ }^{\gamma \delta} H_{\beta \gamma}{ }^{\epsilon} H_{\delta}^{\zeta \iota} H_{\epsilon \zeta \iota} \nabla^{\beta} \nabla^{\alpha} \Phi \\
& +c_{31} H_{\gamma \delta}{ }^{\zeta} H^{\gamma \delta \epsilon} R_{\alpha \epsilon \beta \zeta} \nabla^{\beta} \nabla^{\alpha} \Phi \\
& +c_{32} R_{\alpha}{ }^{\gamma \delta \epsilon} R_{\beta \delta \gamma \epsilon} \nabla^{\beta} \nabla^{\alpha} \Phi \\
& +c_{33} H_{\alpha}{ }^{\gamma \delta} H_{\gamma}{ }^{\epsilon \zeta} R_{\beta \in \delta \zeta} \nabla^{\beta} \nabla^{\alpha} \Phi \\
& +c_{34} H_{\alpha}{ }^{\gamma \delta} H_{\beta}{ }^{\epsilon \zeta} R_{\gamma \epsilon \delta \zeta} \nabla^{\beta} \nabla^{\alpha} \Phi \\
& +c_{35} H_{\alpha}{ }^{\delta \epsilon} \nabla^{\alpha} \Phi \nabla^{\beta} \Phi \nabla_{\gamma} H_{\beta \delta \epsilon} \nabla^{\gamma} \Phi \\
& +c_{36} \nabla_{\alpha} \Phi \nabla^{\alpha} \Phi \nabla_{\beta} \Phi \nabla^{\beta} \Phi \nabla_{\gamma} \Phi \nabla^{\gamma} \Phi \\
& +c_{37} \nabla_{\alpha} \Phi \nabla^{\alpha} \Phi \nabla^{\beta} \Phi \nabla_{\gamma} \nabla_{\beta} \Phi \nabla^{\gamma} \Phi \\
& +c_{38} H_{\beta}{ }^{\delta \epsilon} H_{\gamma \delta \epsilon} \nabla^{\alpha} \Phi \nabla^{\beta} \Phi \nabla^{\gamma} \nabla_{\alpha} \Phi \\
& +c_{39} H_{\beta}{ }^{\delta \epsilon} H_{\gamma \delta \epsilon} \nabla^{\beta} \nabla^{\alpha} \Phi \nabla^{\gamma} \nabla_{\alpha} \Phi \\
& +c_{40} \nabla^{\alpha} \Phi \nabla^{\beta} \Phi \nabla_{\gamma} \nabla_{\beta} \Phi \nabla^{\gamma} \nabla_{\alpha} \Phi \\
& +c_{41} \nabla^{\beta} \nabla^{\alpha} \Phi \nabla_{\gamma} \nabla_{\beta} \Phi \nabla^{\gamma} \nabla_{\alpha} \Phi \\
& +c_{42} H_{\beta}{ }^{\delta \epsilon} H_{\gamma \delta \epsilon} \nabla_{\alpha} \Phi \nabla^{\alpha} \Phi \nabla^{\gamma} \nabla^{\beta} \Phi \\
& +c_{43} H_{\alpha}{ }^{\delta \epsilon} \nabla^{\alpha} \Phi \nabla_{\gamma} H_{\beta \delta \epsilon} \nabla^{\gamma} \nabla^{\beta} \Phi \\
& +c_{44} \nabla_{\alpha} \Phi \nabla^{\alpha} \Phi \nabla_{\gamma} \nabla_{\beta} \Phi \nabla^{\gamma} \nabla^{\beta} \Phi \\
& +c_{45} H_{\alpha \gamma}{ }^{\epsilon} H_{\beta \delta \epsilon} \nabla^{\alpha} \Phi \nabla^{\beta} \Phi \nabla^{\delta} \nabla^{\gamma} \Phi \\
& +c_{46} R_{\alpha \gamma \beta \delta} \nabla^{\alpha} \Phi \nabla^{\beta} \Phi \nabla^{\delta} \nabla^{\gamma} \Phi \\
& +c_{47} H_{\alpha \gamma}{ }^{\epsilon} H_{\beta \delta \epsilon} \nabla^{\beta} \nabla^{\alpha} \Phi \nabla^{\delta} \nabla^{\gamma} \Phi \\
& +c_{48} R_{\alpha \gamma \beta \delta} \nabla^{\beta} \nabla^{\alpha} \Phi \nabla^{\delta} \nabla^{\gamma} \Phi \\
& +c_{49} H_{\beta}{ }^{\delta \epsilon} \nabla^{\alpha} \Phi \nabla^{\gamma} \nabla^{\beta} \Phi \nabla_{\epsilon} H_{\alpha \gamma \delta} \\
& +c_{50} H^{\gamma \delta \epsilon} \nabla^{\alpha} \Phi \nabla^{\beta} \nabla_{\alpha} \Phi \nabla_{\epsilon} H_{\beta \gamma \delta} \\
& +c_{51} \nabla^{\alpha} \Phi \nabla^{\beta} \Phi \nabla_{\delta} H_{\beta \gamma \epsilon} \nabla^{\epsilon} H_{\alpha}{ }^{\gamma \delta} \\
& +c_{52} \nabla^{\beta} \nabla^{\alpha} \Phi \nabla_{\delta} H_{\beta \gamma \epsilon} \nabla^{\epsilon} H_{\alpha}{ }^{\gamma \delta} \\
& +c_{53} \nabla^{\alpha} \Phi \nabla^{\beta} \Phi \nabla_{\epsilon} H_{\beta \gamma \delta} \nabla^{\epsilon} H_{\alpha}{ }^{\gamma \delta} \\
& +c_{54} \nabla^{\beta} \nabla^{\alpha} \Phi \nabla_{\epsilon} H_{\beta \gamma \delta} \nabla^{\epsilon} H_{\alpha}{ }^{\gamma \delta} \\
& +c_{55} \nabla_{\alpha} \Phi \nabla^{\alpha} \Phi \nabla_{\epsilon} H_{\beta \gamma \delta} \nabla^{\epsilon} H^{\beta \gamma \delta} \\
& +c_{56} H_{\alpha}{ }^{\beta \gamma} R_{\gamma \zeta \delta \epsilon} \nabla^{\alpha} \Phi \nabla^{\zeta} H_{\beta}{ }^{\delta \epsilon} \\
& +c_{57} H_{\beta \gamma}{ }^{\epsilon} H^{\beta \gamma \delta} H_{\delta}{ }^{\zeta \iota} \nabla^{\alpha} \Phi \nabla_{\iota} H_{\alpha \epsilon \zeta} \\
& +c_{58} H_{\alpha}{ }^{\beta \gamma} H_{\delta \epsilon}{ }^{\imath} H^{\delta \epsilon \zeta} \nabla^{\alpha} \Phi \nabla_{\iota} H_{\beta \gamma \zeta} \\
& +c_{59} H_{\alpha}{ }^{\beta \gamma} H_{\beta}{ }^{\delta \epsilon} H_{\delta}{ }^{\zeta \iota} \nabla^{\alpha} \Phi \nabla_{\iota} H_{\gamma \epsilon \zeta} \\
& +c_{60} H_{\alpha}{ }^{\beta \gamma} H_{\beta}{ }^{\delta \epsilon} H_{\gamma}{ }^{\zeta \iota} \nabla^{\alpha} \Phi \nabla_{\iota} H_{\delta \epsilon \zeta}
\end{aligned}
$$

where $c_{1}, \ldots, c_{60}$ are 60 parameters which can not be fixed by the gauge invariance constraint.

Up to this point, the above couplings are valid for any higher derivative theory which includes metric, $B$-field and dilaton. In the string theory, however, the parameters in (2),
(3) and (6) may be fixed by imposing some other specific constraints which are valid only in the string theory. For example, one may construct the appropriate S-matrix elements with the above couplings and then compare them with the $\alpha^{\prime}$-expansion of the corresponding sphere-level S-matrix elements in the string theory to fix the parameters. This method has been used in [1] to find the parameters in (2), (3). The parameters $c_{1}, c_{2}$ in (6) have been also found by the $S$-matrix method in [30]. The S-matrix method for fixing all parameters in (6), however, requires one to calculate six-point function in string theory in full details which has not been done yet.

Instead of comparing the S-matrix elements of above couplings with the corresponding S-matrix elements in the string theory, one may impose some other symmetries of the string theory to fix the parameters in (2), (3) and (6). The bosonic string theory has the global T-duality symmetry as well as the gauge symmetries that have been used to find the couplings in (2), (3) and (6). It has been shown in [27] that the T-duality symmetry can fix correctly the couplings in (2), (3) up to overall factors at each order of $\alpha^{\prime}$. In the next section, we show that imposing the T-duality on the couplings in (6) can also fix all 60 parameters in terms of the overall factor at order $\alpha^{\prime}$.

\section{T-duality invariance constraint}

The T-duality constraint on the $D$-dimensional effective action $\mathbf{S}_{\text {eff }}$, in the most simple form, is to reduce the theory on a circle with $U(1)$ isometry to find the $(D-1)$ dimensional effective action $S_{\text {eff }}(\psi)$ where $\psi$ represents all massless fields in the $(D-1)$-dimensional base space. Then one has to transform it under the T-duality transformations to produce $S_{\text {eff }}\left(\psi^{\prime}\right)$ where $\psi^{\prime}$ represents the T-duality transformations of the massless fields in the base space. The T-duality invariance constraint is then

$$
S_{\mathrm{eff}}(\psi)-S_{\mathrm{eff}}\left(\psi^{\prime}\right)=\int d^{D-1} x \sqrt{-\bar{g}} \nabla_{a}\left(e^{-2 \bar{\phi}} J^{a}\right)
$$

where $\bar{g}_{a b}, \bar{\phi}$ are the metric and dilaton in the base space, and $J^{a}$ is an arbitrary covariant vector made of the $(D-1)$ dimensional fields. It has the following $\alpha^{\prime}$-expansion:

$J^{a}=\sum_{n=0}^{\infty} \alpha^{\prime n} J_{n}^{a}$

where $J_{n}^{a}$ is an arbitrary covariant vector at order $\alpha^{\prime n}$.

To have a background with $U(1)$ isometry, it is convenient to use the following background for the metric, $B$-field and dilaton:

$$
G_{\mu \nu}=\left(\begin{array}{cc}
\bar{g}_{a b}+e^{\varphi} g_{a} g_{b} & e^{\varphi} g_{a} \\
e^{\varphi} g_{b} & e^{\varphi}
\end{array}\right),
$$




$$
\begin{aligned}
B_{\mu \nu} & =\left(\begin{array}{cc}
\bar{b}_{a b}+\frac{1}{2} b_{a} g_{b}-\frac{1}{2} b_{b} g_{a} & b_{a} \\
-b_{b} & 0
\end{array}\right), \\
\Phi & =\bar{\phi}+\varphi / 4
\end{aligned}
$$

where $\bar{b}_{a b}$ is the B-field in the base space, and $g_{a}, b_{b}$ are two vectors in this space. Inverse of the above $D$-dimensional metric is

$G^{\mu \nu}=\left(\begin{array}{cc}\bar{g}^{a b} & -g^{a} \\ -g^{b} & e^{-\varphi}+g_{c} g^{c}\end{array}\right)$

where $\bar{g}^{a b}$ is the inverse of the base metric which raises the index of the vectors.

The T-duality transformations at the leading order of $\alpha^{\prime}$ on the $(D-1)$-dimensional fields are given by the Buscher rules $[20,21]$. In the above parametrisation, they become the following linear transformations:

$$
\begin{aligned}
\varphi^{\prime} & =-\varphi, g_{\mu}^{\prime}=b_{\mu}, b_{\mu}^{\prime}=g_{\mu}, \bar{g}_{\alpha \beta}^{\prime}=\bar{g}_{\alpha \beta}, \bar{b}_{\alpha \beta}^{\prime} \\
& =\bar{b}_{\alpha \beta}, \bar{\phi}^{\prime}=\bar{\phi}
\end{aligned}
$$

They form a $Z_{2}$-group, i.e., $\left(x^{\prime}\right)^{\prime}=x$ where $x$ is any field in the base space. At higher orders of $\alpha^{\prime}$, the above transformations receive higher derivative corrections, i.e.,

$\psi^{\prime}=\sum_{n=0}^{\infty} \alpha^{\prime n} \psi_{n}^{\prime}$

where $\psi_{0}^{\prime}$ is the Buscher rules (13), $\psi_{1}^{\prime}$ contains corrections to the Buscher rules at order $\alpha^{\prime}$ and so on. The deformed transformations must satisfy the $Z_{2}$-group.

\subsection{T-duality constraint at orders $\alpha^{\prime 0}, \alpha^{\prime}$}

To impose the constraint (9) on the diffeomorphism invariant couplings (6), we first review how imposing this constraint on the couplings at orders $\alpha^{\prime 0}$ and $\alpha^{\prime}$ can fix their parameters [27]. The constraint (9) at order $\alpha^{\prime 0}$ is

$S_{0}(\psi)-S_{0}\left(\psi_{0}^{\prime}\right)=\int d^{D-1} x \sqrt{-\bar{g}} \nabla_{a}\left(e^{-2 \bar{\phi}} J_{0}^{a}\right)$

where $J_{0}^{a}$ is an arbitrary vector at the leading order of $\alpha^{\prime}$, and $\psi_{0}^{\prime}$ is the Buscher rules (13). Reduction of different scalar terms in $\mathbf{S}_{0}$ are the following [27]:

$$
\begin{aligned}
e^{-2 \Phi} \sqrt{-G} & =e^{-2 \bar{\phi}} \sqrt{-\bar{g}} \\
a_{1} R & =a_{1}\left(\bar{R}-\nabla^{a} \nabla_{a} \varphi-\frac{1}{2} \nabla_{a} \varphi \nabla^{a} \varphi-\frac{1}{4} e^{\varphi} V^{2}\right) \\
a_{2} \nabla_{\mu} \Phi \nabla^{\mu} \Phi & =a_{2}\left(\nabla_{a} \bar{\phi} \nabla^{a} \bar{\phi}+\frac{1}{2} \nabla_{a} \bar{\phi} \nabla^{a} \varphi+\frac{1}{16} \nabla_{a} \varphi \nabla^{a} \varphi\right) \\
a_{3} H^{2} & =a_{3}\left(\bar{H}_{a b c} \bar{H}^{a b c}+3 e^{-\varphi} W^{2}\right)
\end{aligned}
$$

where $V_{a b}$ is field strength of the $U(1)$ gauge field $g_{a}$, i.e., $V_{a b}=\nabla_{a} g_{b}-\nabla_{b} g_{a}$, and $W_{\mu \nu}$ is field strength of the $U(1)$ gauge field $b_{a}$, i.e., $W_{a b}=\nabla_{a} b_{v}-\nabla_{b} b_{a}$. The three-form $\bar{H}$ is defined as $\bar{H}_{a b c}=\tilde{H}_{a b c}-g_{a} W_{b c}-g_{c} W_{a b}-g_{b} W_{c a}$ where the three-form $\tilde{H}$ is field strength of the two-form $\bar{b}_{a b}+\frac{1}{2} b_{a} g_{b}-\frac{1}{2} b_{b} g_{a}$ in (11). The three-form $\bar{H}$ is invariant under the Buscher rules and is not the field strength of a two-form. It satisfies the following Bianchi identity [24]:

$\nabla_{[a} \bar{H}_{b c d]}=-\frac{3}{2} V_{[a b} W_{c d]}$

which is invariant under the Buscher rules (13).

The transformations of different terms in (16) under the Buscher rules (13) can easily be found. Then the T-duality constraint (15) fixes the parameters $a_{1}, a_{2}, a_{3}$ in the $D$ dimensional action [27], i.e.,

$$
\begin{aligned}
\mathbf{S}_{0}= & -\frac{2 a_{1}}{\kappa^{2}} \int d^{D} x e^{-2 \Phi} \sqrt{-G} \\
& \times\left(R+4 \nabla_{a} \Phi \nabla^{a} \Phi-\frac{1}{12} H^{2}\right) .
\end{aligned}
$$

which is the standard effective action at order $\alpha^{\prime 0}$, up to an overall factor. The overall factor must be $a_{1}=1$ to be the effective action of string theory. The constraint (15) fixes also the form of vector $J_{0}^{a}$ in which we are not interested.

The constraint (9) at order $\alpha^{\prime}$ is

$$
\begin{aligned}
& S_{0}(\psi)+\alpha^{\prime} S_{1}(\psi)-S_{0}\left(\psi_{0}^{\prime}+\alpha^{\prime} \psi_{1}^{\prime}\right)-\alpha^{\prime} S_{1}\left(\psi_{0}^{\prime}\right) \\
& =\int d^{D-1} x \sqrt{-\bar{g}} \nabla_{a}\left[e^{-2 \bar{\phi}}\left(J_{0}^{a}+\alpha^{\prime} J_{1}^{a}\right)\right]
\end{aligned}
$$

where $J_{1}^{a}$ is an arbitrary vector at order of $\alpha^{\prime}$, and $\psi_{0}^{\prime}+\alpha^{\prime} \psi_{1}^{\prime}$ is the Buscher rule plus its deformation at order $\alpha^{\prime}$, i.e.,

$$
\begin{aligned}
\varphi^{\prime} & =-\varphi+\alpha^{\prime} \Delta \varphi^{(1)}, g_{a}^{\prime}=b_{a}+\alpha^{\prime} e^{\varphi / 2} \Delta g_{a}^{(1)}, \\
b_{a}^{\prime} & =g_{a}+\alpha^{\prime} e^{-\varphi / 2} \Delta b_{a}^{(1)}, \\
\bar{g}_{a b}^{\prime} & =\bar{g}_{a b}+\alpha^{\prime} \Delta \bar{g}_{a b}^{(1)}, \\
\bar{H}_{a b c}^{\prime} & =\bar{H}_{a b c}+\alpha^{\prime} \Delta \bar{H}_{a b c}^{(1)}, \bar{\phi}^{\prime}=\bar{\phi}+\alpha^{\prime} \Delta \bar{\phi}^{(1)}
\end{aligned}
$$

where $\Delta \varphi^{(1)}, \ldots, \Delta \bar{\phi}^{(1)}$ contains some contractions of $\nabla \varphi, \nabla \bar{\phi}, e^{\varphi / 2} V, e^{-\varphi / 2} W, \bar{H}, \bar{R}$ and their covariant derivatives at order $\alpha^{\prime}$. Since the constraint (19) should have terms up to order $\alpha^{\prime}$, in expanding $S_{0}\left(\psi_{0}^{\prime}+\alpha^{\prime} \psi_{1}^{\prime}\right)$, one should keep the terms up to order $\alpha^{\prime}$, i.e.,

$S_{0}\left(\psi_{0}^{\prime}+\alpha^{\prime} \psi_{1}^{\prime}\right)=S_{0}\left(\psi_{0}^{\prime}\right)+\alpha^{\prime} \delta S_{0}^{(1)}+\cdots$

Using this expansion and the constraint (15), one can simplify the constraint (19) to the following constraint which is only at order $\alpha^{\prime}$ :

$$
\begin{aligned}
& S_{1}(\psi)-S_{1}\left(\psi_{0}^{\prime}\right)-\delta S_{0}^{(1)} \\
& \quad=\int d^{D-1} x \sqrt{-\bar{g}} \nabla_{a}\left[e^{-2 \bar{\phi}} J_{1}^{a}\right]
\end{aligned}
$$

It has been shown in [27] that the above constraint can fix the parameters in the effective action (3) as well as the parameters in the corrections to the Buscher rules up to an overall factor. The result is that $\mathcal{L}_{1}^{2}$ is zero and all terms in $\mathcal{L}_{1}^{1}$ are non-zero, i.e., 


$$
\begin{aligned}
\mathbf{S}_{1}= & \frac{-2 b_{1}}{\kappa^{2}} \alpha^{\prime} \int d^{D} x e^{-2 \Phi} \sqrt{-G} \\
& \times\left(R_{\alpha \beta \gamma \delta} R^{\alpha \beta \gamma \delta}-\frac{1}{2} H_{\alpha}{ }^{\delta \epsilon} H^{\alpha \beta \gamma} R_{\beta \gamma \delta \epsilon}\right. \\
& +\frac{1}{24} H_{\epsilon \delta \zeta} H^{\epsilon}{ }_{\alpha}^{\beta} H^{\delta}{ }_{\beta}^{\gamma} H^{\zeta}{ }^{\alpha} \\
& \left.-\frac{1}{8} H_{\alpha \beta}{ }^{\delta} H^{\alpha \beta \gamma} H_{\gamma}{ }^{\epsilon \zeta} H_{\delta \epsilon \zeta}\right)
\end{aligned}
$$

Up to the overall factor $b_{1}$, the above couplings are the standard effective action of the string theory which has been found in [1] by the S-matrix calculations. For the bosonic string theory $b_{1}=1 / 4$, for the heterotic theory $b_{1}=1 / 8$ and for the superstring theory $b_{1}=0$.

The corrections to the Buscher rules corresponding to the above action are the following [27]:

$$
\begin{aligned}
\Delta \bar{g}_{a b}^{(1)}= & 2 b_{1}\left(e^{\varphi} V_{a}^{c} V_{b c}-e^{-\varphi} W_{a}^{c} W_{b c}\right) \\
\Delta \bar{\phi}^{(1)}= & \frac{b_{1}}{2}\left(e^{\varphi} V^{2}-e^{-\varphi} W^{2}\right) \\
\Delta \varphi^{(1)}= & 2 b_{1}\left(\nabla_{a} \varphi \nabla^{a} \varphi+e^{\varphi} V^{2}+e^{-\varphi} W^{2}\right) \\
\Delta g_{a}^{(1)}= & b_{1}\left(2 e^{-\varphi / 2} \nabla^{b} W_{a b}+e^{\varphi / 2} \bar{H}_{a b c} V^{b c}\right. \\
& \left.-4 e^{-\varphi / 2} \nabla^{b} \bar{\phi} W_{a b}\right) \\
\Delta b_{a}^{(1)}= & -b_{1}\left(2 e^{\varphi / 2} \nabla^{b} V_{a b}+e^{-\varphi / 2} \bar{H}_{a b c} W^{b}\right. \\
& \left.-4 e^{\varphi / 2} \nabla^{b} \bar{\phi} V_{a b}\right) \\
\Delta \bar{H}_{a b c}^{(1)}= & 12 b_{1} \nabla_{[a}\left(W_{b}{ }^{d} V_{c d]}\right)-3 e^{\varphi / 2} V_{[a b} \Delta g_{c]}^{(1)} \\
& -3 e^{-\varphi / 2} W_{[a b} \Delta b_{c]}^{(1)}
\end{aligned}
$$

Replacing these corrections into (20), one finds the corresponding T-duality transformations at order $\alpha^{\prime}$ satisfy the $Z_{2}$-group as well as the Bianchi identity (17) [27]. The constraint (22) fixes also the vector $J_{1}^{a}$ in which we are not interested.

\subsection{T-duality constraint at order $\alpha^{\prime 2}$}

We now study in details the constraint (9) at order $\alpha^{\prime 2}$ to fix the 60 parameters in (6). This constraint at order $\alpha^{\prime 2}$ is

$$
\begin{aligned}
& S_{0}(\psi)+\alpha^{\prime} S_{1}(\psi)+\alpha^{\prime 2} S_{2}(\psi)-S_{0}\left(\psi_{0}^{\prime}\right. \\
& \left.\quad+\alpha^{\prime} \psi_{1}^{\prime}+\alpha^{\prime 2} \psi_{2}^{\prime}\right)-\alpha^{\prime} S_{1}\left(\psi_{0}^{\prime}+\alpha^{\prime} \psi_{1}^{\prime}\right)-\alpha^{\prime 2} S_{2}\left(\psi_{0}^{\prime}\right) \\
& =\int d^{D-1} x \sqrt{-\bar{g}} \nabla_{a}\left[e^{-2 \bar{\phi}}\left(J_{0}^{a}+\alpha^{\prime} J_{1}^{a}+\alpha^{\prime 2} J_{2}^{a}\right)\right]
\end{aligned}
$$

where $J_{2}^{a}$ is an arbitrary vector at order $\alpha^{\prime 2}, \alpha^{\prime} \psi_{1}^{\prime}$ represents the corrections to the Buscher rules at order $\alpha^{\prime}$, e.g., (24), and $\alpha^{\prime 2} \psi_{2}^{\prime}$ represents the corrections to the Buscher rules at order $\alpha^{\prime 2}$, i.e.,

$$
\varphi^{\prime}=-\varphi+\alpha^{\prime} \Delta \varphi^{(1)}+\frac{1}{2} \alpha^{\prime 2} \Delta \varphi^{(2)},
$$

$$
\begin{aligned}
g_{a}^{\prime} & =b_{a}+\alpha^{\prime} e^{\varphi / 2} \Delta g_{a}^{(1)}+\frac{1}{2} \alpha^{\prime 2} e^{\varphi / 2} \Delta g_{a}^{(2)} \\
b_{a}^{\prime} & =g_{a}+\alpha^{\prime} e^{-\varphi / 2} \Delta b_{a}^{(1)}+\frac{1}{2} \alpha^{\prime 2} e^{-\varphi / 2} \Delta b_{a}^{(2)}, \\
\bar{g}_{a b}^{\prime} & =\bar{g}_{a b}+\alpha^{\prime} \Delta \bar{g}_{a b}^{(1)}+\frac{1}{2} \alpha^{\prime 2} \Delta \bar{g}_{a b}^{(2)} \\
\bar{H}_{a b c}^{\prime} & =\bar{H}_{a b c}+\alpha^{\prime} \Delta \bar{H}_{a b c}^{(1)}+\frac{1}{2} \alpha^{\prime 2} \Delta \bar{H}_{a b c}^{(2)} \\
\bar{\phi}^{\prime} & =\bar{\phi}+\alpha^{\prime} \Delta \bar{\phi}^{(1)}+\frac{1}{2} \alpha^{\prime 2} \Delta \bar{\phi}^{(2)}
\end{aligned}
$$

where $\Delta \varphi^{(2)}, \ldots, \Delta \bar{\phi}^{(2)}$ contains all contractions of $\nabla \varphi$, $\nabla \bar{\phi}, e^{\varphi / 2} V, e^{-\varphi / 2} W, \bar{H}, \bar{R}$ and their covariant derivatives at order $\alpha^{\prime 2}$ with unknown coefficients. Since the constraint (25) should have terms up to order $\alpha^{\prime 2}$, in expanding $S_{0}\left(\psi_{0}^{\prime}+\alpha^{\prime} \psi_{1}^{\prime}+\alpha^{\prime 2} \psi_{2}^{\prime}\right)$ and $\alpha^{\prime} S_{1}\left(\psi_{0}^{\prime}+\alpha^{\prime} \psi_{1}^{\prime}\right)$, one should keep the terms up to order $\alpha^{\prime 2}$, i.e.,

$$
\begin{aligned}
S_{0}\left(\psi_{0}^{\prime}+\alpha^{\prime} \psi_{1}^{\prime}+\alpha^{\prime 2} \psi_{2}^{\prime}\right)= & S_{0}\left(\psi_{0}^{\prime}\right)+\alpha^{\prime} \delta S_{0}^{(1)} \\
& +\alpha^{\prime 2} \delta S_{0}^{(2)}+\cdots \\
\alpha^{\prime} S_{1}\left(\psi_{0}^{\prime}+\alpha^{\prime} \psi_{1}^{\prime}\right)= & \alpha^{\prime} S_{1}\left(\psi_{0}^{\prime}\right)+\alpha^{\prime 2} \delta S_{1}^{(1)}+\cdots
\end{aligned}
$$

Using these expansions and the constraints (15) and (22), one can simplify the constraint (25) to the following constraint which is only at order $\alpha^{\prime 2}$ :

$$
\begin{aligned}
& S_{2}(\psi)-S_{2}\left(\psi_{0}^{\prime}\right)-\delta S_{0}^{(2)}-\delta S_{1}^{(1)} \\
& =\int d^{D-1} x \sqrt{-\bar{g}} \nabla_{a}\left[e^{-2 \bar{\phi}} J_{2}^{a}\right]
\end{aligned}
$$

The speculation is that this constraint as well as the constraint that the T-duality transformations should satisfy the $Z_{2}$-group and the anomalous Bianchi identity (17) may fix all parameters in the diffiomorphism invariant couplings (6).

The T-duality transformations (26) should satisfy the $Z_{2}$ group. This produces the following constraint between the corrections at orders $\alpha^{\prime}$ and $\alpha^{\prime 2}$ :

$$
\begin{array}{r}
-\Delta \varphi^{(2)}(\psi)+\Delta \varphi^{(2)}\left(\psi_{0}^{\prime}\right)+2 \delta \Delta \varphi^{(1)}\left(\psi_{0}^{\prime}\right)=0 \\
\Delta b_{a}^{(2)}(\psi)+\Delta g_{a}^{(2)}\left(\psi_{0}^{\prime}\right)+2 \delta \Delta g_{a}^{(1)}\left(\psi_{0}^{\prime}\right)=0 \\
\Delta g_{a}^{(2)}(\psi)+\Delta b_{a}^{(2)}\left(\psi_{0}^{\prime}\right)+2 \delta \Delta b_{a}^{(1)}\left(\psi_{0}^{\prime}\right)=0 \\
\Delta \bar{g}_{a b}^{(2)}(\psi)+\Delta \bar{g}_{a b}^{(2)}\left(\psi_{0}^{\prime}\right)+2 \delta \Delta \bar{g}_{a b}^{(1)}\left(\psi_{0}^{\prime}\right)=0 \\
\Delta \bar{H}_{a b c}^{(2)}(\psi)+\Delta \bar{H}_{a b c}^{(2)}\left(\psi_{0}^{\prime}\right)+2 \delta \Delta \bar{H}_{a b c}^{(1)}\left(\psi_{0}^{\prime}\right)=0 \\
\Delta \bar{\phi}^{(2)}(\psi)+\Delta \bar{\phi}^{(2)}\left(\psi_{0}^{\prime}\right)+2 \delta \Delta \bar{\phi}^{(1)}\left(\psi_{0}^{\prime}\right)=0
\end{array}
$$

where we have used the $Z_{2}$-constraint at order $\alpha^{\prime}$ which are [27]

$$
\begin{array}{r}
-\Delta \varphi^{(1)}(\psi)+\Delta \varphi^{(1)}\left(\psi_{0}^{\prime}\right)=0 \\
\Delta b_{a}^{(1)}(\psi)+\Delta g_{a}^{(1)}\left(\psi_{0}^{\prime}\right)=0 \\
\Delta g_{a}^{(1)}(\psi)+\Delta b_{a}^{(1)}\left(\psi_{0}^{\prime}\right)=0 \\
\Delta \bar{g}_{a b}^{(1)}(\psi)+\Delta \bar{g}_{a b}^{(1)}\left(\psi_{0}^{\prime}\right)=0 \\
\Delta \bar{H}_{a b c}^{(1)}(\psi)+\Delta \bar{H}_{a b c}^{(1)}\left(\psi_{0}^{\prime}\right)=0
\end{array}
$$




$$
\Delta \bar{\phi}^{(1)}(\psi)+\Delta \bar{\phi}^{(1)}\left(\psi_{0}^{\prime}\right)=0
$$

The perturbations $\delta \Delta \varphi^{(1)}\left(\psi_{0}^{\prime}\right), \ldots, \delta \Delta \bar{\phi}^{(1)}\left(\psi_{0}^{\prime}\right)$ in (29) are defined as

$$
\begin{aligned}
& \Delta \varphi^{(1)}\left(\psi_{0}^{\prime}+\alpha^{\prime} \psi_{1}^{\prime}\right) \\
& \quad=\Delta \varphi^{(1)}\left(\psi_{0}^{\prime}\right)+\alpha^{\prime} \delta \Delta \varphi^{(1)}\left(\psi_{0}^{\prime}\right)+\cdots \\
& e^{\varphi / 2}\left(\psi_{0}^{\prime}+\alpha^{\prime} \psi_{1}^{\prime}\right) \Delta g_{a}^{(1)}\left(\psi_{0}^{\prime}+\alpha^{\prime} \psi_{1}^{\prime}\right) \\
& \quad=e^{-\varphi / 2} \Delta g_{a}^{(1)}\left(\psi_{0}^{\prime}\right)+\alpha^{\prime} e^{-\varphi / 2} \delta \Delta g_{a}^{(1)}\left(\psi_{0}^{\prime}\right)+\cdots \\
& e^{-\varphi / 2}\left(\psi_{0}^{\prime}+\alpha^{\prime} \psi_{1}^{\prime}\right) \Delta b_{a}^{(1)}\left(\psi_{0}^{\prime}+\alpha^{\prime} \psi_{1}^{\prime}\right) \\
& \quad=e^{\varphi / 2} \Delta b_{a}^{(1)}\left(\psi_{0}^{\prime}\right)+\alpha^{\prime} e^{\varphi / 2} \delta \Delta b_{a}^{(1)}\left(\psi_{0}^{\prime}\right)+\cdots \\
& \Delta \bar{g}_{a b}^{(1)}\left(\psi_{0}^{\prime}+\alpha^{\prime} \psi_{1}^{\prime}\right) \\
& =\Delta \bar{g}_{a b}^{(1)}\left(\psi_{0}^{\prime}\right)+\alpha^{\prime} \delta \Delta \bar{g}_{a b}^{(1)}\left(\psi_{0}^{\prime}\right)+\cdots \\
& \Delta \bar{H}_{a b c}^{(1)}\left(\psi_{0}^{\prime}+\alpha^{\prime} \psi_{1}^{\prime}\right) \\
& =\Delta \bar{H}_{a b c}^{(1)}\left(\psi_{0}^{\prime}\right)+\alpha^{\prime} \delta \Delta \bar{H}_{a b c}^{(1)}\left(\psi_{0}^{\prime}\right)+\cdots \\
& \Delta \bar{\phi}^{(1)}\left(\psi_{0}^{\prime}+\alpha^{\prime} \psi_{1}^{\prime}\right) \\
& =\Delta \bar{\phi}^{(1)}\left(\psi_{0}^{\prime}\right)+\alpha^{\prime} \delta \Delta \bar{\phi}^{(1)}\left(\psi_{0}^{\prime}\right)+\cdots
\end{aligned}
$$

where dots represent the perturbations at higher orders of $\alpha^{\prime}$ which do not appear in our calculations.

The Bianchi identity (17) in terms of 3-form $H$ and 1forms $g, b$ is $d H=-(3 / 2) d g \wedge d b$. The T-dual fields should satisfy this identity as well, i.e.,

$$
\begin{aligned}
& d\left(\bar{H}+\alpha^{\prime} \Delta \bar{H}^{(1)}+\frac{1}{2} \alpha^{\prime 2} \Delta \bar{H}^{(2)}+\cdots\right) \\
& =-\frac{3}{2} d\left(b+\alpha^{\prime} e^{\varphi / 2} \Delta g^{(1)}+\frac{1}{2} \alpha^{\prime 2} e^{\varphi / 2} \Delta g^{(2)}+\cdots\right) \\
& \wedge d\left(g+\alpha^{\prime} e^{-\varphi / 2} \Delta b^{(1)}+\frac{1}{2} \alpha^{\prime 2} e^{-\varphi / 2} \Delta b^{(2)}+\cdots\right)
\end{aligned}
$$

This relation at order $\alpha^{\prime 0}$ gives the Bianchi identity (17). At order $\alpha^{\prime}$ it gives the following relation between the corrections to the Buscher rules at order $\alpha^{\prime}$ :

$$
\Delta \bar{H}^{(1)}=\tilde{H}^{(1)}-\frac{3}{2}\left[d b \wedge\left(e^{-\varphi / 2} \Delta b^{(1)}\right)+\left(e^{\varphi / 2} \Delta g^{(1)}\right) \wedge d g\right]
$$

where $\tilde{H}^{(1)}$ is a $U(1) \times U(1)$ invariant closed 3-form, i.e., $d \tilde{H}^{(1)}=0$, at order $\alpha^{\prime}$ which is odd under parity. The corrections (24) satisfy this relation. At order $\alpha^{\prime 2}$, the Bianchi identity (32) produces the following relation between the corrections at orders $\alpha^{\prime}$ and $\alpha^{\prime 2}$ :

$$
\begin{aligned}
\Delta \bar{H}^{(2)}= & \tilde{H}^{(2)}-\frac{3}{2}\left[d b \wedge\left(e^{-\varphi / 2} \Delta b^{(2)}\right)+\left(e^{\varphi / 2} \Delta g^{(2)}\right) \wedge d g\right. \\
& +\left(e^{\varphi / 2} \Delta g^{(1)}\right) \wedge d\left(e^{-\varphi / 2} \Delta b^{(1)}\right) \\
& \left.+d\left(e^{\varphi / 2} \Delta g^{(1)}\right) \wedge\left(e^{-\varphi / 2} \Delta b^{(1)}\right)\right]
\end{aligned}
$$

where $\tilde{H}^{(2)}$ is a closed 3-form, i.e., $d \tilde{H}^{(2)}=0$, which contains all contractions of $\nabla \varphi, \nabla \bar{\phi}, e^{\varphi / 2} V, e^{-\varphi / 2} W, \bar{H}, \bar{R}$ and their covariant derivatives at order $\alpha^{\prime 2}$ with unknown coefficients.

Therefore, the second order corrections $\Delta \varphi^{(2)}$, $\Delta g_{a}^{(2)}, \Delta b_{a}^{(2)}, \Delta \bar{g}_{a b}^{(2)}$ and $\Delta \bar{\phi}^{(2)}$ should be all contractions of $\nabla \varphi, \nabla \bar{\phi}, e^{\varphi / 2} V, e^{-\varphi / 2} W, \bar{H}, \bar{R}$ and their covariant derivatives at order $\alpha^{\prime 2}$ with unknown coefficients. The correction $\Delta \bar{H}_{a b c}^{(2)}$ is then can be calculated from (34). All corrections should satisfy the $Z_{2}$-relations (29). They produce some algebraic equations between the parameters of the corrections at order $\alpha^{\prime 2}$ and the parameter $b_{1}$ in the corrections at order $\alpha^{\prime}$, i.e., (24). These parameters and the 60 parameters in the action (6) should satisfy the constraint (28) as well.

To use the constraint (28) one needs to reduce the couplings in (6). The reduction of each term at order $\alpha^{\prime 2}$ is a very lengthy expression. However, the final result for the reduction of each term must be an invariant term under the $U(1) \times U(1)$ gauge transformations. Using this fact as a constraint, the calculations of the reduction of $\mathbf{S}_{2}$ can be simplified greatly. The couplings in (6) have only Riemann curvature, $H, \nabla H$, $\nabla \Phi$ and $\nabla \nabla \Phi$. So we need to reduce these terms and then contract them with the metric (12). In the reduction of these terms, there are many terms which contains gauge field $g_{a}$ without its field strength. These terms must be cancelled at the end of the day for the scalar couplings. Hence, to simplify the calculation we drop those terms in the reduction of $R_{\mu \nu \alpha \beta}, H_{\mu \nu \alpha}, \nabla_{\mu} H_{\nu \alpha \beta}, \nabla_{\mu} \Phi, \nabla_{\mu} \nabla_{\nu} \Phi$ and $G^{\mu \nu}$ which have the gauge field $g_{a}$. Using this simplification, the reduction of Riemann curvature becomes ${ }^{1}$

$$
\begin{aligned}
& R_{a b c d}=\bar{R}_{a b c d}+\frac{1}{4} e^{\varphi}\left(V_{a d} V_{b c}-V_{a c} V_{b d}-2 V_{a b} V_{c d}\right) \\
& R_{a b c y}=\frac{1}{4} e^{\varphi}\left(V_{b c} \nabla_{a} \varphi-V_{a c} \nabla_{b} \varphi-2 V_{a b} \nabla_{c} \varphi-2 \nabla_{c} V_{a b}\right) \\
& R_{a y c y}=\frac{1}{4} e^{\varphi}\left(e^{\varphi} V_{a}{ }^{b} V_{c b}-\nabla_{a} \varphi \nabla_{c} \varphi-2 \nabla_{c} \nabla_{a} \varphi\right)
\end{aligned}
$$

All other components are either zero or related to the above terms by the Riemann symmetries. The reduction of different components of $\nabla \nabla \Phi$ and $\nabla \Phi$ become

$$
\begin{aligned}
\nabla_{a} \nabla_{b} \Phi & =\nabla_{a} \nabla_{b} \bar{\phi}+\frac{1}{4} \nabla_{a} \nabla_{b} \varphi \\
\nabla_{a} \nabla_{y} \Phi & =-\frac{1}{2} e^{\varphi}\left(V_{a b} \nabla^{b} \bar{\phi}+\frac{1}{4} V_{a b} \nabla^{b} \varphi\right) \\
\nabla_{y} \nabla_{y} \Phi & =\frac{1}{2} e^{\varphi}\left(\nabla_{a} \varphi \nabla^{a} \bar{\phi}+\frac{1}{4} \nabla_{a} \varphi \nabla^{a} \varphi\right) \\
\nabla_{a} \Phi & =\nabla_{a} \bar{\phi}+\frac{1}{4} \nabla_{a} \varphi ; \quad \nabla_{y} \Phi=0
\end{aligned}
$$

The reduction of different components of $\nabla H$ and $H$ become

$$
\begin{aligned}
\nabla_{a} H_{b c d} & =\frac{1}{2}\left(V_{a d} W_{b c}-V_{a c} W_{b d}+V_{a b} W_{c d}+2 \nabla_{a} \bar{H}_{b c d}\right. \\
\nabla_{a} H_{b c y} & =\frac{1}{2}\left(e^{\varphi} \bar{H}_{b c d} V_{a}{ }^{d}-W_{b c} \nabla_{a} \varphi+2 \nabla_{a} W_{b c}\right) \\
\nabla_{y} H_{b c d} & =\frac{1}{2} e^{\varphi}\left(\bar{H}_{b d a} V_{c}{ }^{a}-\bar{H}_{c d a} V_{b}{ }^{a}-\bar{H}_{b c a} V_{d}{ }^{a}\right)
\end{aligned}
$$

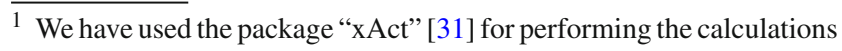
in this paper. 


$$
\begin{aligned}
& +\frac{1}{2}\left(W_{b d} \nabla_{c} \varphi-W_{c d} \nabla_{b} \varphi-W_{b c} \nabla_{d} \varphi\right) \\
\nabla_{y} H_{b c y}= & \frac{1}{2} e^{\varphi}\left(\bar{H}_{b c a} \nabla^{a} \varphi-V_{c}{ }^{a} W_{b a}+V_{b}{ }^{a} W_{c a}\right) \\
H_{a b c}= & \bar{H}_{a b c} ; \quad H_{a b y}=W_{a b}
\end{aligned}
$$

The covariant derivatives on the right-hand side of (35), (36) and (37) are $(D-1)$-dimensional, and the indices are raised by the inverse metric $\bar{g}^{a b}$. The reduction of inverse of the $D$-dimensional metric in this case also becomes

$G^{\mu \nu}=\left(\begin{array}{cc}\bar{g}^{a b} & 0 \\ 0 & e^{-\varphi}\end{array}\right)$

Using above reductions, one can calculate the reduction of different scalar terms in (6).

Then using the constraint (28), one finds some equations involving the 60 parameters in (6), the arbitrary parameters of $J_{2}^{a}$ and the parameters of $\Delta \varphi^{(2)}, \Delta g_{a}^{(2)}, \Delta b_{a}^{(2)}, \Delta \bar{g}_{a b}^{(2)}$, $\Delta \bar{\phi}^{(2)}$ and $\tilde{H}^{(2)}$. The parameters of $\Delta \varphi^{(2)}, \Delta g_{a}^{(2)}, \Delta b_{a}^{(2)}$, $\Delta \bar{g}_{a b}^{(2)}, \Delta \bar{\phi}^{(2)}$ and $\tilde{H}^{(2)}$ should also satisfy the constraints (29) and (34). To solve these constraints, one has to write the couplings in them in terms of independent couplings. Then coefficients of the independent couplings which involve the above parameters should be zero. To perform this last steps, one has to impose the following Bianchi identities into the constraints as well:

$$
\begin{aligned}
\bar{R}_{a[b c d]} & =0, \\
\nabla_{[a} \bar{R}_{b c] d e} & =0 \\
{[\nabla, \nabla] \mathcal{O} } & =\bar{R} \mathcal{O} \\
\nabla_{[a} V_{b c]} & =0 \\
\nabla_{[a} W_{b c]} & =0 \\
\nabla_{[a} \bar{H}_{b c d]}+\frac{3}{2} V_{[a b} W_{c d]} & =0
\end{aligned}
$$

To impose the last identity above, we contract it with tensors $\nabla \varphi, \nabla \bar{\phi}, e^{\varphi / 2} V, e^{-\varphi / 2} W, \bar{H}, \bar{R}$ and their derivatives with arbitrary parameters and then add them to the constraints. To impose the first three identities above, we use the locally inertial frame in which these identities are automatically satisfied. In the locally inertial frame, the metric $\bar{g}_{a b}$ takes its canonical form and its first derivatives are all vanish, i.e., ,

$\bar{g}_{a b}=\eta_{a b}, \quad \partial_{a} \bar{g}_{b c}=0$

The second and higher derivatives of metric, however, are non-zero. In this coordinate, by rewriting the covariant derivative in terms of partial derivatives, one finds the first three identities in (39) are satisfied. To satisfy the Bianchi identities $d V=0=d W$ as well, in the couplings which involve derivatives of $V$ and $W$, we rewrite them in terms of their gauge fields, i.e., $V_{a b}=\partial_{a} g_{b}-\partial_{b} g_{a}$ and $W_{a b}=$ $\partial_{a} b_{b}-\partial_{b} b_{a}$.

After using the above steps to write the couplings in the constraints (28), (29) and (34) in terms of independent cou- plings in the local frame, one can set their coefficients to zero to produce some algebraic equations involving only the parameters. Interestingly, these algebraic equations fix all the 60 parameters in (6) in terms of $b_{1}$, the overall factor at order $\alpha^{\prime}$ which should be $b_{1}=1 / 4$ for the bosonic string theory. All 20 parameters in (7) are non-zero and only 7 parameters in (8) are non-zero. They are

$$
\begin{aligned}
& \mathbf{S}_{2}=\frac{-2 b_{1}}{\kappa^{2}} \alpha^{2} \int d^{D} x e^{-2 \Phi} \sqrt{-G} \\
& \times\left(-\frac{1}{12} H_{\alpha}{ }^{\delta \epsilon} H^{\alpha \beta \gamma} H_{\beta \delta^{\zeta}} H_{\gamma}{ }^{\iota \kappa} H_{\epsilon \iota}{ }^{\mu} H_{\zeta \kappa \mu}\right. \\
& +\frac{1}{30} H_{\alpha \beta}{ }^{\delta} H^{\alpha \beta \gamma} H_{\gamma}{ }^{\epsilon \zeta} H_{\delta}{ }^{i \kappa} H_{\epsilon \zeta}{ }^{\mu} H_{\iota \kappa \mu} \\
& +\frac{3}{10} H_{\alpha \beta}{ }^{\delta} H^{\alpha \beta \gamma} H_{\gamma}{ }^{\epsilon \zeta} H_{\delta \epsilon}{ }^{l} H_{\zeta}{ }^{\kappa \mu} H_{l \kappa \mu} \\
& +\frac{13}{20} H_{\alpha}{ }^{\epsilon \zeta} H_{\beta}{ }^{\imath \kappa} H_{\gamma \epsilon \zeta} H_{\delta \iota \kappa} R^{\alpha \beta \gamma \delta} \\
& +\frac{2}{5} H_{\alpha}{ }^{\epsilon \zeta} H_{\beta \epsilon}{ }^{\iota} H_{\gamma \zeta}{ }^{\kappa} H_{\delta \iota \kappa} R^{\alpha \beta \gamma \delta} \\
& +\frac{18}{5} H_{\alpha \gamma}{ }^{\epsilon} H_{\beta}{ }^{\zeta \iota} H_{\delta \zeta}{ }^{\kappa} H_{\epsilon \iota \kappa} R^{\alpha \beta \gamma \delta} \\
& -\frac{43}{5} H_{\alpha \gamma}{ }^{\epsilon} H_{\beta}{ }^{\zeta \iota} H_{\delta \epsilon}{ }^{\kappa} H_{\zeta \iota \kappa} R^{\alpha \beta \gamma \delta} \\
& -\frac{16}{5} H_{\alpha \gamma}{ }^{\epsilon} H_{\beta \delta^{\zeta}} H_{\epsilon}{ }^{{ }^{\kappa}} H_{\zeta \iota \kappa} R^{\alpha \beta \gamma \delta} \\
& -2 H_{\beta \epsilon}{ }^{\iota} H_{\delta \zeta \iota} R_{\alpha}{ }^{\epsilon} \gamma^{\zeta} R^{\alpha \beta \gamma \delta}-2 H_{\beta \delta}{ }^{\imath} H_{\epsilon \zeta \iota} R_{\alpha}{ }^{\epsilon} \gamma^{\zeta} R^{\alpha \beta \gamma \delta} \\
& -\frac{4}{3} R_{\alpha}{ }^{\epsilon} \gamma^{\zeta} R^{\alpha \beta \gamma \delta} R_{\beta \zeta \delta \epsilon}+\frac{4}{3} R_{\alpha \beta}{ }^{\epsilon \zeta} R^{\alpha \beta \gamma \delta} R_{\gamma \epsilon \delta \zeta} \\
& +3 H_{\beta}{ }^{\zeta \iota} H_{\epsilon \zeta \iota} R^{\alpha \beta \gamma \delta} R_{\gamma}{ }^{\epsilon}{ }_{\alpha \delta} \\
& +2 H_{\beta \epsilon}{ }^{\iota} H_{\delta \zeta \iota} R^{\alpha \beta \gamma \delta} R_{\gamma}{ }^{\epsilon}{ }^{\zeta}+2 H_{\alpha \beta \epsilon} H_{\delta \zeta \iota} R^{\alpha \beta \gamma \delta} R_{\gamma}{ }^{\epsilon \zeta \iota} \\
& +\frac{13}{10} H_{\alpha}{ }^{\gamma \delta} H_{\beta \gamma}{ }^{\epsilon} H_{\delta}{ }^{\zeta \iota} H_{\epsilon \zeta \iota} \nabla^{\beta} \nabla^{\alpha} \Phi \\
& +\frac{13}{5} H_{\gamma}{ }^{\epsilon \zeta} H_{\delta \epsilon \zeta} R_{\alpha}{ }^{\gamma}{ }^{\delta} \nabla^{\beta} \nabla^{\alpha} \Phi \\
& -\frac{52}{5} H_{\beta \delta^{\zeta}} H_{\gamma \epsilon \zeta} R_{\alpha}{ }^{\gamma \delta \epsilon} \nabla^{\beta} \nabla^{\alpha} \Phi \\
& -\frac{26}{5} H_{\alpha \gamma \epsilon} H_{\beta \delta \zeta} R^{\gamma \delta \epsilon \zeta} \nabla^{\beta} \nabla^{\alpha} \Phi \\
& +\frac{13}{5} \nabla^{\beta} \nabla^{\alpha} \Phi \nabla_{\epsilon} H_{\beta \gamma \delta} \nabla^{\epsilon} H_{\alpha}{ }^{\gamma \delta} \\
& +\frac{13}{10} H_{\beta \gamma}{ }^{\epsilon} H^{\beta \gamma \delta} H_{\delta}{ }^{\zeta \iota} \nabla^{\alpha} \Phi \nabla_{\iota} H_{\alpha \epsilon \zeta} \\
& -\frac{13}{20} H_{\alpha}{ }^{\beta \gamma} H_{\delta \epsilon}{ }^{\iota} H^{\delta \epsilon \zeta} \nabla^{\alpha} \Phi \nabla_{\iota} H_{\beta \gamma \zeta} \\
& +\frac{1}{20} H_{\alpha}{ }^{\delta \epsilon} H^{\alpha \beta \gamma} \nabla_{\iota} H_{\delta \epsilon \zeta} \nabla^{\iota} H_{\beta \gamma}{ }^{\zeta} \\
& +\frac{1}{5} H_{\alpha}{ }^{\delta \epsilon} H^{\alpha \beta \gamma} \nabla_{\zeta} H_{\gamma \epsilon \iota} \nabla^{\iota} H_{\beta \delta^{\zeta}} \\
& -\frac{6}{5} H_{\alpha}{ }^{\delta \epsilon} H^{\alpha \beta \gamma} \nabla_{\iota} H_{\gamma \epsilon \zeta} \nabla^{\iota} H_{\beta \delta^{\zeta}} \\
& -\frac{6}{5} H_{\alpha \beta}{ }^{\delta} H^{\alpha \beta \gamma} \nabla_{\zeta} H_{\delta \epsilon \iota} \nabla^{\iota} H_{\gamma}{ }^{\epsilon \zeta}
\end{aligned}
$$




$$
\left.+\frac{17}{10} H_{\alpha \beta}{ }^{\delta} H^{\alpha \beta \gamma} \nabla_{\iota} H_{\delta \epsilon \zeta} \nabla^{\iota} H_{\gamma}{ }^{\epsilon \zeta}\right)
$$

Note that the 60 parameters are fixed when the Bianchi constraint (34) is imposed as well as the T-duality constraint (28) and (29). If one only uses the constraint (28) and (29), then 12 parameters of (6) remain arbitrary. It is the constraint (34) which fixes these 12 parameters as well.

The algebraic equations also fix some of the parameters in the T-duality transformations and the parameters of total derivative terms at order $\alpha^{\prime 2}$ in terms of $b_{1}$, and leave many of them to be arbitrary. Some of the arbitrary parameters in the T-duality transformations may be removed by the Bianchi identities and some of them are related to the coordinate transformations at order $\alpha^{\prime 2}$. Even when all the arbitrary parameters are set to zero, there are still too may terms in the T-duality transformations at order $\alpha^{\prime 2}$, so we do not write them explicitly. On the other hand, those corrections are only needed if one would like to extend the above couplings to the order $\alpha^{\prime 3}$ in the bosonic theory in which we are not interested in this paper. The important part of the calculations is that there are 60 relations between the 60 parameters in (6) and the parameter $b_{1}$, i.e., the T-duality constraint fixes all 60 parameters at order $\alpha^{\prime 2}$ in terms of the overall factor of the couplings at order $\alpha^{\prime}$ ! This ends our illustration of the fact that the T-duality constraint on the effective action can fix uniquely the effective action of bosonic string theory at order $\alpha^{\prime 2}$.

\section{Discussion}

In this paper, we have shown that imposing the gauge symmetries and the T-duality symmetry on the effective action of string theory for metric, $B$-field and dilaton at order $\alpha^{\prime 2}$, can fix the effective action, i.e., (40), up to an overall factor which is the overall factor of the effective action at order $\alpha^{\prime}$. This is extension of the similar calculation at order $\alpha^{\prime}$ done in [27] which fixes the effective action at order $\alpha^{\prime}$ up to the overall factor $b_{1}$, i.e., (23). In fact, the gauge symmetries require to have 60 couplings at order $\alpha^{\prime 2}$ with unfixed coefficients [2], and the T-duality symmetry which is imposed on the reduction of the effective action on a circle, fixes these 60 parameters.

In the base space, we have done the calculations in the local frame in which the first derivatives of the base metric is zero. After solving the constraints, we have imposed the solution for the parameters in the constraints (28), (29), (34) and found that they are satisfied even when the first derivative of metric is non-zero. It is as expected, because the constraints are some covariant identities. If they satisfy in one particular frame like the local inertial frame, they would satisfy in all other frames as well.
Most of the couplings in (40) are new couplings which have not been found in the literature by other methods in string theory. When $B$-field is zero, the couplings (40) reduce to two Riemann cubed terms that their coefficients, after using the cyclic symmetry of the Riemann curvature, become exactly the same as the coefficients that have been found in [30] by the S-matrix method. These couplings are invariant under the field redefinitions. However, the couplings which have $B$-field are not invariant under the field redefinitions. When $B$-field is non-zero, one may check the couplings involving four fields with the corresponding four-point $S$ matrix elements in bosonic string theory. To check this comparison, one has to use a field redefinition that change the Riemann squared terms in (23) to the Gauss-Bonnet combination in which the propagators do not receive $\alpha^{\prime}$-correction. That field redefinitions would then change the form of the couplings in (40). The resulting couplings then may be checked with the corresponding S-matrix elements. We leave the details of this calculation for the future works.

We have found that seven dilaton couplings in (8) are nonzero. On the other hand, it is known that the couplings at order $\alpha^{\prime 2}$ depends on the effective action at order $\alpha^{\prime}$ [28]. We have used the minimal action (23) and the corresponding T-duality transformations (24). Using another scheme for the couplings at order $\alpha^{\prime}$, some of the parameters in (40) may be changed. It would be interesting to check if there is a scheme for the couplings at order $\alpha^{\prime}$ for which all the dilaton couplings in (8) become zero.

We have found the effective action (40) by imposing only the symmetries of string theory, i.e., the $B$-field gauge invariance, diffeomorphism and T-duality invariances. As a result, the effective action (40) is background independent. However, the total derivative terms are ignored in imposing the T-duality constraint. Hence the effective action (40) is valid for all backgrounds that have no boundary. It would be interesting to take into account in details the total derivative terms to find the boundary terms as well as the bulk terms for the general case that the background has boundary.

We have done the calculations in the curved base space to make sure that the constraints (28), (29), (34) are satisfied in full details. We have performed the calculations in flat base space as well and found exactly the same parameters for (6) as in (40). In the T-duality calculations at order $\alpha^{\prime}$ [27] which have correctly reproduced the effective action at order $\alpha^{\prime}$, it is also assumed that the base space is flat. Hence, for the calculations at the higher orders of $\alpha^{\prime}$ which would be very lengthy calculations, one may safely assume the base space is flat. The most simple calculations at order $\alpha^{\prime 3}$ is for superstring theory in which the T-duality transformations have no deformation at orders $\alpha^{\prime}$ and $\alpha^{\prime 2}$. It would be interesting to perform this calculations at order $\alpha^{13}$ in the superstring to find the $B$-field couplings which are not known in the literature. 
If one extends the calculations in the bosonic theory to the order $\alpha^{\prime 3}$, one would find a set of couplings which are proportional to $b_{1}$ and another set of couplings that their overall factor is arbitrary. The comparison with the four-point $\mathrm{S}$ matrix elements dictates that this factor should be $\zeta(3)$. At order $\alpha^{\prime 4}$, again one should find a set of couplings which are proportional to $b_{1}$, a set of couplings proportial to $\zeta(3)$ and some other sets of couplings that their overall factor may be fixed by the corresponding S-matrix elements. Continuing these logic, one would find sets of couplings at each order of $\alpha^{\prime}$ which are proportional to $b_{1}$. Hence, one expects the T-duality constraint produces a set of couplings at each order of $\alpha^{\prime}$ that are proportional to $b_{1}$. They form a complete set of couplings which would be invariant under the T-duality transformations at all orders of $\alpha^{\prime}$. That T-dual set of couplings may have de Sitter solution [32]. It would be interesting to find this T-dual set.

In this paper, while we have deformed the T-duality transformations, we have assumed the gauge transformations are the standard diffeomorphisms and $B$-field gauge transformations which are the correct transformations in the bosonic and superstring theories. In the superstring theory $b_{1}=0$, hence, the couplings (40) are zero in the superstring theory as expected. On the other hand, the 60 parameters in (40) do not dependent on the dimension of spacetime. That does not indicate the result (40) is valid also for the heterotic theory for $b_{1}=1 / 8$. The reason is that in the heterotic theory the $B$-field gauge transformation is deformed at order $\alpha^{\prime}$ which is resulted from the Green-Schwarz anomaly cancellation mechanism [33]. To produce the heterotic result, one has to add to the couplings (6) the fixed couplings at order $\alpha^{\prime 2}$ which are resulted from the deformed gauge transformations, i.e., $-\frac{\alpha^{\prime 2}}{12} \Omega_{\mu \nu \alpha} \Omega^{\mu \nu \alpha}$ where $\Omega$ is the three-form Chern-Simons which can be written in terms of spin connection,

$$
\begin{aligned}
\Omega_{\mu \nu \alpha} & =\omega_{[\mu i}{ }^{j} \partial_{\nu} \omega_{\alpha] j}{ }^{i}+\frac{2}{3} \omega_{[\mu i}{ }^{j} \omega_{\nu j}{ }^{k} \omega_{\alpha] k}{ }^{i} ; \\
\omega_{\mu i}{ }^{j} & =\partial_{\mu} e_{\beta}{ }^{j} e^{\beta}{ }_{i}-\Gamma_{\mu \beta}{ }^{\gamma} e_{\gamma}{ }^{j} e^{\beta}{ }_{i}
\end{aligned}
$$

where $e_{\mu}{ }^{i} e_{\nu}{ }^{j} \eta_{i j}=G_{\mu \nu}$. Adding this term, the corresponding T-duality transformations and the 60 parameters in (6) may be fixed in the heterotic theory. We didn't perform this calculations, however, one expects all parameters in (6) to be zero. Similar calculation at order $\alpha^{\prime}$ has been done in [27]. The T-duality structure of the couplings in the heterotic theory has been studied in [34-38] in the DFT formalism.

There is another deformation of $B$-field gauge transformations which correspond to the Chiral string theory [39]. The deformation at order $\alpha^{\prime}$ is the same as the deformation in the heterotic theory in which spin connection is replaced by the Christoffel connection [40]. The low energy effective action of this theory at the leading order, is given by the T-duality invariant action (18) and at the order $\alpha^{\prime}$, it is given by the T-duality invariant coupling $H^{\mu \nu \alpha} \boldsymbol{\Omega}_{\mu \nu \alpha}[41]^{2}$ where the three-form Chern-Simons $\boldsymbol{\Omega}_{\mu \nu \alpha}$ is resulted from the deformed gauge transformation, i.e.,

$\boldsymbol{\Omega}_{\mu \nu \alpha}=\Gamma_{[\mu \beta} \partial_{\nu} \Gamma_{\alpha] \gamma}^{\beta}+\frac{2}{3} \Gamma_{\left[\mu \beta^{\gamma}\right.} \Gamma_{\nu \gamma}^{\lambda} \Gamma_{\alpha] \lambda}^{\beta}$

To find the effective action at order $\alpha^{\prime 2}$, one has to add to the couplings (6) the fixed couplings at order $\alpha^{\prime 2}$, i.e., $-\frac{\alpha^{\prime 2}}{12} \boldsymbol{\Omega}_{\mu \nu \alpha} \boldsymbol{\Omega}^{\mu \nu \alpha}$. Adding this term, the corresponding Tduality transformations and the 60 parameters in (6) may be fixed in the Chiral string theory by the T-duality constraint method. It would be interesting to perform this calculation to find the $\alpha^{\prime 2}$-order terms. This theory has been studied in the DFT formalism in $[42,43]$.

In general, both the diffeomorphisms and the $B$-field gauge transformations may receive higher derivative deformations in a general gauge invariant higher-derivative theory. One may impose these gauge transformations and the deformed T-duality transformations to study the effective action of a higher-derivative theory which is invariant under the gauge transformations and under the T-duality transformations. The effective action at the leading order of $\alpha^{\prime}$ is given by (18). At order $\alpha^{\prime}$, the parity invariant part of the effective action would be more general than the action (23). It would be interesting to find this effective action.

Acknowledgements This work is supported by Ferdowsi University of Mashhad under grant 1/50251(1398/04/31).

Data Availability Statement This manuscript has no associated data or the data will not be deposited. [Authors' comment: The results in this paper are obtained analytically, hence, it does not use any data.]

Open Access This article is distributed under the terms of the Creative Commons Attribution 4.0 International License (http://creativecomm ons.org/licenses/by/4.0/), which permits unrestricted use, distribution, and reproduction in any medium, provided you give appropriate credit to the original author(s) and the source, provide a link to the Creative Commons license, and indicate if changes were made. Funded by SCOAP ${ }^{3}$.

\section{References}

1. R.R.Metsaev, A.A. Tseytlin, Nucl. Phys. B 293, 385 (1987). https:// doi.org/10.1016/0550-3213(87)90077-0

2. M.R. Garousi, H. Razaghian. arXiv:1905.10800 [hep-th]

3. J. Scherk, J.H. Schwarz, Phys. Lett. 52B, 347 (1974). https://doi. org/10.1016/0370-2693(74)90059-8

4. T. Yoneya, Prog. Theor. Phys. 51, 1907 (1974). https://doi.org/10. 1143/PTP.51.1907

5. C.G. Callan Jr., E.J. Martinec, M.J. Perry, D. Friedan, Nucl. Phys. B 262, 593 (1985). https://doi.org/10.1016/0550-3213(85)90506-1

6. E.S. Fradkin, A.A. Tseytlin, Phys. Lett. 158B, 316 (1985). https:// doi.org/10.1016/0370-2693(85)91190-6

$\overline{2 \text { The parameter }} b_{1}$, in the parity invariant action (23), corresponding to the Chiral string is $b_{1}=0$. 
7. E.S. Fradkin, A.A. Tseytlin, Phys. Lett. 160B, 69 (1985). https:// doi.org/10.1016/0370-2693(85)91468-6

8. A. Giveon, M. Porrati, E. Rabinovici, Phys. Rept. 244, 77 (1994). https://doi.org/10.1016/0370-1573(94)90070-1. arXiv:hep-th/9401139

9. E. Alvarez, L. Alvarez-Gaume, Y. Lozano, Nucl. Phys. Proc. Suppl. 41, 1 (1995). https://doi.org/10.1016/0920-5632(95)00429-D. arXiv:hep-th/9410237

10. W. Siegel, Phys. Rev. D 47, 5453 (1993). https://doi.org/10.1103/ PhysRevD.47.5453. arXiv:hep-th/9302036

11. W. Siegel, Phys. Rev. D 48, 2826 (1993). https://doi.org/10.1103/ PhysRevD.48.2826. arXiv:hep-th/9305073

12. W. Siegel, Manifest duality in low-energy superstrings. arXiv:hep-th/9308133

13. C. Hull, B. Zwiebach, JHEP 0909, 099 (2009). https://doi.org/10. 1088/1126-6708/2009/09/099. arXiv:0904.4664 [hep-th]

14. G. Aldazabal, D. Marques, C. Nunez, Class. Quant. Grav. 30, 163001 (2013). https://doi.org/10.1088/0264-9381/30/16/ 163001. arXiv:1305.1907 [hep-th]

15. O. Hohm, C. Hull, B. Zwiebach, JHEP 1008, 008 (2010). https:// doi.org/10.1007/JHEP08(2010). arXiv:1006.4823 [hep-th]

16. O. Hohm, B. Zwiebach, JHEP 1411, 075 (2014). https://doi.org/ 10.1007/JHEP11(2014). arXiv:1407.3803 [hep-th]

17. D. Marques, C.A. Nunez, JHEP 1510, 084 (2015). https://doi.org/ 10.1007/JHEP10(2015). arXiv:1507.00652 [hep-th]

18. M.R. Garousi, Phys. Rev. D 98(6), 066008 (2018). https://doi.org/ 10.1103/PhysRevD.98.066008. arXiv:1805.08977 [hep-th]

19. M.R. Garousi, Phys. Rept. 702, 1 (2017). https://doi.org/10.1016/ j.physrep.2017.07.009. arXiv:1702.00191 [hep-th]

20. T.H. Buscher, Phys. Lett. B 194, 59 (1987). https://doi.org/10. 1016/0370-2693(87)90769-6

21. T.H. Buscher, Phys. Lett. B 201, 466 (1988). https://doi.org/10. 1016/0370-2693(88)90602-8

22. A.A. Tseytlin, Mod. Phys. Lett. A 6, 1721 (1991). https://doi.org/ 10.1142/S021773239100186X

23. E. Bergshoeff, B. Janssen, T. Ortin, Class. Quant. Grav. 13, 321 (1996). https://doi.org/10.1088/0264-9381/13/3/002. arXiv:hep-th/9506156

24. N. Kaloper, K.A. Meissner, Phys. Rev. D 56, 7940 (1997). https:// doi.org/10.1103/PhysRevD.56.7940. arXiv:hep-th/9705193

25. H. Razaghian, M.R. Garousi, JHEP 1802, 056 (2018). https://doi. org/10.1007/JHEP02(2018). arXiv:1709.01291 [hep-th]

26. H. Razaghian, M.R. Garousi, Phys. Rev. D 97, 106013 (2018). https://doi.org/10.1103/PhysRevD.97.106013. arXiv:1801.06834 [hep-th]
27. M.R. Garousi, Phys. Rev. D 99(12), 126005 (2019). https://doi. org/10.1103/PhysRevD.99.126005. arXiv:1904.11282 [hep-th]

28. M.C. Bento, O. Bertolami, J.C. Romao, Phys. Lett. B 252, 401 (1990). https://doi.org/10.1016/0370-2693(90)90559-O

29. K.A. Meissner, Phys. Lett. B 392, 298 (1997). https://doi.org/10. 1016/S0370-2693(96)01556-0. arXiv:hep-th/9610131

30. R.R. Metsaev, A.A. Tseytlin, Phys. Lett. B 185, 52 (1987). https:// doi.org/10.1016/0370-2693(87)91527-9

31. T. Nutma, Comput. Phys. Commun. 185, 1719 (2014). https://doi. org/10.1016/j.cpc.2014.02.006. arXiv:1308.3493 [cs.SC]

32. O. Hohm, B. Zwiebach. arXiv:1905.06963 [hep-th]

33. M.B. Green, J.H. Schwarz, Phys. Lett. 149B, 117 (1984). https:// doi.org/10.1016/0370-2693(84)91565-X

34. O.A. Bedoya, D. Marques, C. Nunez, JHEP 1412, 074 (2014). https://doi.org/10.1007/JHEP12(2014)074. arXiv:1407.0365 [hep-th]

35. A. Coimbra, R. Minasian, H. Triendl, D. Waldram, JHEP 1411, 160 (2014). https://doi.org/10.1007/JHEP11(2014)160. arXiv: 1407.7542 [hep-th]

36. K. Lee, Nucl. Phys. B 899, 594 (2015). https://doi.org/10.1016/j. nuclphysb.2015.08.013. arXiv:1504.00149 [hep-th]

37. W.H. Baron, J.J. Fernandez-Melgarejo, D. Marques, C. Nunez, JHEP 1704, 078 (2017). https://doi.org/10.1007/ JHEP04(2017)078. arXiv:1702.05489 [hep-th]

38. W.H. Baron, E. Lescano, D. Marqués, JHEP 1811, 160 (2018). https://doi.org/10.1007/JHEP11(2018)160. arXiv:1810.01427 [hep-th]

39. O. Hohm, W. Siegel, B. Zwiebach, JHEP 1402, 065 (2014). https:// doi.org/10.1007/JHEP02(2014)065. arXiv:1306.2970 [hep-th]

40. O. Hohm, B. Zwiebach, JHEP 1501, 012 (2015). https://doi.org/ 10.1007/JHEP01(2015)012. arXiv:1407.0708 [hep-th]

41. O. Hohm, B. Zwiebach, JHEP 1604, 101 (2016). https://doi.org/ 10.1007/JHEP04(2016)101. arXiv:1510.00005 [hep-th]

42. O. Hohm, B. Zwiebach, Phys. Rev. D 93(6), 064035 (2016). https://doi.org/10.1103/PhysRevD.93.064035. arXiv:1509.02930 [hep-th]

43. E. Lescano, D. Marques, JHEP 1706, 104 (2017). https://doi.org/ 10.1007/JHEP06(2017)104. arXiv:1611.05031 [hep-th] 